\title{
Performance of the preliminary definition of improvement in juvenile chronic arthritis patients treated with methotrexate
}

Nicolino Ruperto, Angelo Ravelli, Fernanda Falcini, Loredana Lepore, Rosetta De Sanctis, Francesco Zulian, Antonella Buoncompagni, Maria Luisa Sardella, Ciro Strano, Maria Alessio, Stefania Viola, Alberto Martini, for the Italian Pediatric Rheumatology Study Group

Laboratorio di Informatica Medica, IRCCS S Matteo, Pavia, Italy

N Ruperto

Clinica Pediatrica, IRCCS S Matteo,

Pavia, Italy

A Ravelli

$S$ Viola

A Martini

Clinica Pediatrica, Ospedale Meyer, Florence, Italy

F Falcini

Clinica Pediatrica, Istituto Burlo Garofalo, Trieste, Italy L Lepore

Ospedale Bambin Gesù, Rome, Italy $\mathrm{R}$ De Sanctis

Clinica Pediatrica, Padova, Italy

F Zulian

II Divisione di Pediatria, Istituto Gaslini, Genova, Italy A Buoncompagni

Clinica Pediatrica, Università, Turin, Italy M L Sardella

Clinica Pediatrica, Naples, Italy C Strano

II Divisione di Pediatria, Naples, Italy M Alessio

Correspondence to: Dr A Martini, Clinica Pediatrica, Università di Pavia, IRCCS Policlinico S Matteo, P le Golgi 2, 27100 Pavia, Italy.

Accepted for publication 27 October 1997
Abstract

Objective-To investigate the performance of the core set of outcome measures and the preliminary definition of improvement (PDI) in the assessment of response to methotrexate (MTX) treatment in children with juvenile chronic arthritis (JCA).

Methods-Data were obtained from an open label, non-controlled trial designed to investigate the efficacy of MTX in children with JCA. All patients had the core set of variables assessed at baseline and after six months of treatment. Variables in the core set are: (1) physician global assessment of disease activity; (2) parent or patient (if appropriate in age) global assessment of overall well being; (3) functional ability; (4) number of joints with active arthritis; (5) number of joints with limited range of motion; (6) erythrocyte sedimentation rate. The PDI specifies that to be classified as improved, a patient must show at least $30 \%$ improvement from baseline in three of any six variables in the core set, with no more than one of the remaining variables worsening by more than $30 \%$.

Results-A total of 111 JCA patients were included in the study. According to the PDI, after six months of MTX treatment 73 patients $(66 \%)$ were classified as improved and $38(34 \%)$ as not improved. Among the core set variables, parent assessment detected the highest percentage of patients improved (72\%) and functional assessment the lowest (37\%).

Conclusion-The PDI identifies about two thirds of patients with JCA treated with low dose MTX as improved. This proportion is similar to that expected to improve based upon a previous controlled study of low dose, oral MTX and provides preliminary evidence of the definition's validity.

(Ann Rheum Dis 1998;57:38-41)
In juvenile chronic arthritis (JCA) clinical trials, multiple measures of disease activity are usually assessed and different trials often include different endpoints. Furthermore, the amount of change in each variable that signifies clinically important improvement or worsening is not established. This lack of standardisation can confuse the interpretation of results and makes difficult inter-trial comparisons. ${ }^{1}$ To standardise the assessment of clinical response and increase the efficiency of JCA clinical trials, a core set of outcome variables ${ }^{2}$ and a preliminary definition of improvement (PDI) ${ }^{3}$ for use in children with juvenile arthritis have been recently developed. The clinical validity of the core set and the PDI in children with JCA remains to be investigated in future clinical trials. Retrospective analysis of the PDI is hampered by the lack of published therapeutic trials including all six core set variables. All these variables were included, however, in a recent Italian multicentre trial on oral methotrexate (MTX) in resistant JCA. Using data from this trial, we have evaluated retrospectively the performance of the PDI in the assessment of the clinical response to MTX.

\section{Methods}

PATIENT SELECTION

All patients included in the study were part of an open label, multicentre non-controlled trial designed to investigate the efficacy and incidence of side effects in JCA patients treated with MTX. ${ }^{3 a}$

To be eligible for enrolment, patients had to have: (1) a diagnosis of JCA according to the criteria of the European League Against Rheumatism (EULAR) ${ }^{4}$; (2) a disease duration of at least six months; (3) at least five joints with active arthritis (defined as the presence of swelling or limitation of movement with either pain upon movement or tenderness) that was not adequately controlled by non-steroidal anti-inflammatory drugs or disease modifying antirheumatic drugs (DMARDs). No patient was simultaneously taking DMARDs, and all patients previously treated with DMARDs had stopped taking the drug at least three months 
before starting MTX. No patient was previously given MTX or had received intraarticular corticosteroid injections in the preceding three months. All patients received MTX orally at a dose of $10 \mathrm{mg} / \mathrm{m}^{2} /$ week in a single weekly dose throughout the trial. All centres followed an identical clinical protocol ${ }^{5}$ and used standardised case report forms containing all the core set variables.

CORE SET OF OUTCOME MEASURES AND PRELIMINARY DEFINITION OF IMPROVEMENT IN JCA

Variables included in the core set are the following: (1) physician global assessment of disease activity (MD global) on a $10 \mathrm{~cm}$ visual analogue scale (VAS) anchored by the words "remission" and "very severe"; (2) parent or patient (if appropriate in age) global assessment of overall well being (parent/patient global) on a $10 \mathrm{~cm}$ VAS anchored by the words "very well" and "very poor"; (3) functional ability; (4) number of joints with active arthritis (NJAA); (5) number of joints with limited range of motion (NJLROM); (6) erythrocyte sedimentation rate (ESR). The PDI specifies that, to be classified as improved, a patient must show at least 30\% improvement from baseline in three of any six variables in the core set, with no more than one of the remaining variables worsening by more than $30 \%$.

ASSESSMENT OF THE CORE SET VARIABLES

The six core set variables were assessed in all patients at baseline and after six months of MTX treatment. The MD global and the parent/patient global were measured using different scales than those indicated in the core set. In particular, categorical scales were used instead of visual analogue scales. The MD global was scored on a 5 point ordered categorical scale $(1=$ none, $2=$ mild, $3=$ moder ate, $4=$ severe, $5=$ very severe); the parent/ patient global was assessed by asking parents to judge their child's overall well being at six months as compared with baseline according to a 3 point categorical scale (better, same, worse); patients were not asked to self report their overall well being. The functional ability was measured by using either: (1) the Modified Lee Index ${ }^{6}$; (2) the Juvenile Arthritis Functional Assessment Report (JAFAR) ${ }^{7}$; (3)

Table 1 Values of the core set variables in the 111 patients with $\mathcal{F C A}$ at the beginning and after six months of MTX treatment

\begin{tabular}{lll}
\hline & Baseline & $\begin{array}{l}\text { After six } \\
\text { months }\end{array}$ \\
\hline $\begin{array}{l}\text { MD global (no of patients) } \\
\text { class 1 }\end{array}$ & 0 & 31 \\
class 2 & 0 & 39 \\
class 3 & 36 & 24 \\
$\quad$ class 4 & 50 & 8 \\
$\quad$ class 5 & 25 & 9 \\
Parent global (no of patients) & & \\
better & - & 80 \\
same & - & 20 \\
worse & - & 11 \\
Functional ability (mean (SD)) & $0.7(0.7)$ & $0.3(0.5)$ \\
No of joints with LROM (mean (SD)) & $14(12)$ & $10(12)$ \\
No of joints with active arthritis (mean (SD)) & $15(11)$ & $10(10)$ \\
ESR (mm 1st h) (mean (SD)) & $58(30)$ & $35(28)$ \\
\hline
\end{tabular}

^Parent global assessment of overall well being was not quantified at baseline (see text for explanation). LROM: limited range of motion; ESR: erythrocyte sedimentation rate. the Italian version of the Childhood Health Assessment Questionnaire (CHAQ) disability index. $^{89}$ In most patients the functional assessment was done by using only one of these instruments, whereas others had their functional status evaluated by more than one method. In the event a single patient was assessed by more than one instrument, priority was given to the CHAQ, followed by the JAFAR, and than the Modified Lee Index. CHAQ scores range from $0-3$ with 0 representing the best functional ability and 3 the worst. To standardise scores from all three instruments, scores from the JAFAR and the Modified Lee Index were proportionally converted to the $0-3$ scale of the CHAQ. We previously observed a very high correlation among the three instruments when given to the same patient on the same day. ${ }^{10}$ Thus, we felt justified in combining scores from the different instruments for purposes of analysis. The NJAA and the NJLROM were assessed in 64 joints. The presence of active arthritis was defined as indicated above. The presence of a limited range of motion was defined for each joint as a loss of at least $5^{\circ}$ in any articular movement with respect to the normal amplitude. The ESR was determined by the Westergren method. The assessment of articular variables as well as the physician global assessment were performed in each patient by the same investigator throughout the study.

\section{ASSESSMENT OF CLINICAL RESPONSE}

Response to MTX treatment was evaluated in all patients by comparing the values of the core end points at six months of treatment with the baseline values. Patients were classified as improved or not improved according to the PDI. For the MD global, a decrease in at least two categories from baseline was considered equivalent to the $30 \%$ change required by the PDI. Because of the lack of baseline quantification of the parent global assessment, patients who were judged by their parents as "better", with respect to the baseline condition, were considered as improved by at least $30 \%$.

To avoid small changes from baseline producing undue influence on the classification of the patients' outcome, if the baseline and the six month index for functional ability and ESR were $\leqslant 0.3$ or $\leqslant 15$, respectively, the percental change of both variables was set equal to 0 .

STATISTICAL ANALYSIS

To evaluate the clinical validity of the PDI descriptive statistics were mainly used. The $\chi^{2}$ test was used when proportions where compared. For the issue of redundancy (colinearity) we determined Spearman correlation coefficients ( $r$ values) between actual changes in the six core set variables from baseline to the last visit; evidence of colinearity was considered to be present if the $r$ values was $\geqslant 0.7$. For the parent global we substituted the number -1 for the category better, 0 for same, +1 for worse. Statistical packages used were SAS 
Table 2 Frequency distribution of the outcome of the core set variables after six months of MTX treatment

\begin{tabular}{lll}
\hline Variables & $\begin{array}{l}\text { Improved } \\
n(\%)\end{array}$ & $\begin{array}{l}\text { Not improved } \\
n(\%)\end{array}$ \\
\hline MD global & $59(53)$ & $52(47)$ \\
Parent global & $80(72)$ & $31(28)$ \\
Functional assessment & $41(37)$ & $70(63)$ \\
No of joints with LROM & $61(55)$ & $50(45)$ \\
No of active joints & $67(60)$ & $44(40)$ \\
ESR & $65(59)$ & $46(41)$ \\
\hline
\end{tabular}

^Defined as $\geqslant 30 \%$ improvement from baseline values. Abbreviations as in table 1 .

Version 6.11 for Windows, SAS Institute Inc, Cary, NC, and Excel 5.0 (Microsoft Corporation).

\section{Results}

A total of 111 children with JCA, 74 girls and 37 boys, completed the six month treatment regimen with no drop outs. The age at onset of JCA ranged from 0.5 to 15.3 years (mean 5.5 ) and the disease duration from 0.5 to 14.9 years (mean 3.4). The disease onset subtype was systemic in 40 patients, polyarticular in 43 patients (three rheumatoid factor positive), and pauciarticular in 28 patients. At the time MTX was started all patients had polyarticular involvement. Table 1 shows the values of the core set variables at baseline and after six months. At the end of the six months of treatment, 73 patients $(66 \%)$ were classified as improved and $38(34 \%)$ as not improved, according to the PDI. Among the six core set variables, parent assessment detected the highest percentage of patients improved $(72 \%)$ and functional assessment the lowest (37\%) (table 2).

To evaluate the relative importance of each of the six core set variables, we then eliminated one variable at a time from the outcome computation. The highest decrease in the number of patients classified as improved was observed when the parent global was removed ( $8 \%)$ and the lowest after the elimination of the functional ability (5\%).

Table 3 presents the $r$ values for correlation's among actual (rather than per cent) changes in the core set variables between the baseline and the six month evaluation. There was some evidence of colinearity between the NJAA and the NJLROM.

\section{Discussion}

We investigated retrospectively the performance of the PDI in a cohort of children with JCA treated with low dose MTX. After six months of treatment, the PDI identified two thirds of patients as improved. This percentage of improvement is similar to that reported in a

Table 3 Spearman correlation coefficients between the six core set variables; the actual change between baseline and final evaluations was used for the correlation

\begin{tabular}{lcclll}
\hline & ESR & $\begin{array}{l}\text { Parent } \\
\text { global }\end{array}$ & $\begin{array}{c}\text { MD } \\
\text { global }\end{array}$ & Function & $\begin{array}{l}\text { No of joints } \\
\text { with LROM }\end{array}$ \\
\hline Parent global & 0.27 & & & & \\
MD global & 0.47 & 0.56 & & & \\
Functional ability & 0.24 & 0.25 & 0.51 & 0.23 & 0.70 \\
No of joints with LROM & 0.29 & 0.30 & 0.40 & 0.31 & \\
No of active joints & 0.34 & 0.36 & 0.54 & 0.31
\end{tabular}

Abbreviations as in table 1 . previous controlled study. ${ }^{11}$ When examined separately, however, the six variables of the core set showed a different ability to disclose patient improvement, with parent global assessment of overall well being detecting the highest percentage of patients improved, and functional assessment the lowest. Although the parent assessment was done by a more crude scale than that included in the PDI, this finding suggests that subjective parent judgement represents a responsive instrument in JCA. Subjective measures were found to be reliable responsive variables in controlled trials in adult patients with rheumatoid arthritis. ${ }^{12}$ A possible explanation of the lower performance of the functional assessment is that improvement in it is more objective, probably with less placebo effect, and may also take longer for a statistically significant improvement to become detectable. Of note, the functional ability tool to be used is not specified in the PDI. Nevertheless, the six variables of the core set seemed to be equally important when combined into the PDI, because the elimination of one of them from the computation one at time did not change significantly the overall response rate to MTX treatment. We found no overlapping among the variables except for colinearity, although to the lower threshold level, of active joint count with the number of limited joints. Taken together, these results support the usefulness of all the six variables included in the PDI.

Although the PDI was created to be robust enough to cover all types of JCA, its relative performance in the various subsets of the disease is not established. For example, it is unclear whether the PDI may perform better in children with polyarthritis than in those with fewer joints involved or whether in systemic patients the presence of extraarticular features (particularly fever) may influence the physician's or parent/ patient' subjective opinion of response to treatment. As all our patients had polyarthritis at the time MTX was started, however, our data are of value only for patients with a polyarticular involvement of JCA. Moreover, although many of our systemic patients had ongoing systemic features at baseline, the extraarticular manifestations (such as fever reduction) was not routinely recorded. These issues should be investigated in future validation studies.

We acknowledge that because some of our assessments differ from those of the PDI, this may affect our results and limit our conclusions about the validity of the PDI.

In conclusion, the PDI identifies about two thirds of patients with JCA treated with low dose MTX as improved. This proportion is similar to that expected to improve based upon a previous controlled study of low dose, oral MTX and provides preliminary evidence of the definition's validity. The discriminant ability of the definition in its final proposed form should be further investigated in prospective controlled studies.

This work was supported by IRCCS Policlinico S Matteo, Pavia, Italy. We wish to thank Dr Edward H Giannini, MSc, DrPH (Cincinnati, OH, USA) for his critical review of the manuscript. 
1 Ruperto N, Giannini EH. Redundancy of conventional articular response variables used in juvenile chronic arthritis clinical trials. Ann Rheum Dis 1996;55:73-5.

2 Giannini EH, Lovell DJ, Felson DT, Goldsmith CH. Preliminary core set of outcome variables for use in JRA clinical trials. Arthritis Rheum 1994;37(suppl):S428.

3 Giannini EH, Ruperto N, Ravelli A, Lovell DJ, Felson DT, Martini A. Preliminary definition of improvement in juvenile arthritis. Arthritis Rheum 1997;40:1202-9.

3a Ravelli A, Gerloni V, Corona F, Falcini F, Lepore L, De Sanctis $\mathrm{R}$, et al. Oral versus intramuscular methotrexate in juvenile chronic arthritis. Clin Exp Rheumatol (in press).

4 Wood PHN. Special meeting on: nomenclature and classification of arthritis in children. In: Munthe E, ed. The care of rheumatic children. Basle: EULAR Publishers, 1987:47-50.

5 Ravelli A, Ruperto N, Ramenghi B, Aramini L, Martini A. Protocollo di valutazione del trattamento con methotrexate nell'artrite cronica giovanile (Standardized protocol for the assessment of methotrexate treatment in juvenile chronic
arthritis). Revista Italiana di Pediatria (It J Pediatr) 1994;20:92-5.

6 Ravelli A, Viola S, Ramenghi B, Di Fuccia G, Ruperto N, Zonta L, et al. Evaluation of response to methotrexate by a functional index in juvenile chronic arthritis. Clin Rheumatol $1995 ; 14: 322-6$.

7 Howe S, Levinson J, Shear E, Hartner S, McGirr G, Schulte $\mathrm{M}$, et al. Development of a disability measurement tool for juvenile rheumatoid arthritis. The Juvenile Arthritis Functional Assessment Report for children and their parents. Arthritis Rheum 1991;34:873-80.

8 Singh G, Athreya BH, Fries JF, Goldsmith DP. Measurement of health status in children with juvenile rheumatoid arthritis. Arthritis Rheum 1994;37:1761-9.

9 Fantini F, Corvaglia G, Bergomi P, Gattinara M, Gerloni V, Lomater C, et al. Validation of the Italian version of the Stanford Childhood Health Assessment Questionnaire for measuring functional status in children with chronic arthritis. Clin Exp Rheumatol 1995;13:785-91.

10 Ravelli A, Viola S, Ruperto N, Corsi B, Ballardini G, Martini A. Correlation between conventional disease activity measures in juvenile chronic arthritis. Ann Rheum Dis 1997;56:197-200.

11 Giannini EH, Brewer EJ, Kuzmina N, Shaikov A, Maximov A, Vorontsov I, et al. Methotrexate in resistant juvenile rheumatoid arthritis. Results of the U.S.A.-U.S.S.R. double-blind, placebo-controlled trial. N Engl J Med 1992; 326:1043-9.

12 Buchbinder R, Bombardier C, Yeung M, Tugwell P. Which outcome measures should be used in rheumatoid arthritis clinical trials? Clinical and quality-of-life measures' responsiveness to treatment in a randomized controlled trial. Arthritis Rheum 1995;38:1568-80. 